\title{
URINARY PROTEIN EXCRETION AND RENAL HEMODYNAMIC ADJUSTMENTS DURING ORTHOSTASIS IN PATIENTS WITH ACUTE AND CHRONIC RENAL DISEASES 1, 2
}

\author{
By WILLOUGHBY LATHEM,8 BETTY S. ROOF, JAMES F. NICKEL,4 AND \\ STANLEY E. BRADLEY
}

\author{
(From the Department of Medicine, College of Physicians and Surgeons, Columbia University, \\ and the Presbyterian Hospital, New York, N. Y.)
}

(Submitted for publication March 5, 1954; accepted July 7, 1954)

The amount and character of the protein lost in the urine may vary widely during the course of renal disease (2). Since proteinuria seems to result from increased permeability of the glomerular capillaries $(2,3)$ (though a reduction in tubular reabsorption of protein may also play a role [4] ), variation in proteinuria may be ascribed chiefly to changes in glomerular function. Progression or healing of the renal lesion undoubtedly alters the structure and area of the glomerular filtering surface with resultant change in the escape of protein from the plasma into the urine (5-8). In addition, proteinuria is affected by renal circulatory adjustments that may influence filtration. The renal vascular responses to congestive heart failure, shock, fever, violent exercise or pressor drugs such as epinephrine, are often associated with the appearance of proteinuria in normal persons and with augmentation of proteinuria in patients with renal disease $(5,9-12)$. Less striking changes in the renal circulation, such as those associated with assumption of the upright position, do not usually result in loss of protein by the normal kidney. Pre-existing proteinuria, on the other hand, may increase during orthostasis, suggesting that glomerular permeability may be more susceptible to change in the diseased kidney. The effect of orthostasis upon glomerular filtration, renal blood flow, and protein excretion

\footnotetext{
1 This work was supported by a grant from the Medical Research and Development Board, Office of the Surgeon General, Department of the Army, under contract No. DA-49-007-MD-205.

2 These data have been presented in preliminary form elsewhere (1).

3 Postdoctorate research fellow, Public Health Service, 1951-1952. Present address: Veterans Hospital, West Haven, Connecticut.

4 Life Insurance Medical Research Fund Fellow, 19511952. Present address: Walter Reed Army Medical Center, Washington, D. C.
}

was studied in patients with acute or chronic renal diseases in order to define more accurately the role of renal hemodynamic alterations in producing variation in proteinuria.

\section{METHODS}

Twenty studies were made in 19 fasting patients with well-documented renal disease: Acute diffuse glomerulonephritis (four), chronic diffuse glomerulonephritis (six), benign nephrosclerosis (three), chronic pyelonephritis (two), disseminated lupus erythematosus (two), malignant nephrosclerosis (one), and multiple myeloma without Bence-Jones proteinuria (one). Two subjects (W. M. and M. M.) with chronic diffuse glomerulonephritis also suffered from the nephrotic syndrome. Two studies were performed on one subject (M. M.).

An effort was made to select subjects with marked proteinuria as judged by the heat-acetic acid test. However, since this method provides only a rough estimate of the concentration of urinary protein, some subjects excreting less than four grams of protein per day were included in the series.

The glomerular filtration rate was measured as the inulin clearance, and effective renal plasma flow as the p-aminohippurate ${ }^{5}$ clearance $(9,13)$. Tubular function was assessed in terms of the maximal rate of glucose reabsorption by the tubules (glucose $\left.T_{m}\right)(9,13)$. Urinary protein concentration was determined by the micro-Kjeldahl method (7) and plasma protein concentration in blood collected without stasis by a specific gravity (copper sulfate) method (14). Total protein clearance was calculated from the total excretion (mg. per min.) divided by the total plasma concentration ( $\mathrm{mg}$. per ml.). This calculation is useful in expressing alterations in total protein output relative to the plasma concentration provided changes in individual protein fractions of plasma or urine do not occur. Blood urea nitrogen was measured (15) in all but one subject (J. W.). The blood pressure was measured sphygmomanometrically and the mean pressure calculated as the diastolic pressure plus 40 per cent of the pulse pressure (16).

In seven subjects urinary and serum or plasma protein patterns were determined by paper electrophoresis (17)

\footnotetext{
5 Supplied through the courtesy of Dr. William Boger, Sharpe and Dohme, Inc., Glenolden, Penna.
} 
before and during orthostasis and on return to the horizontal. In an attempt to obtain sufficient protein concentrations for clear resolution and identification, aliquots of pooled urine (usually 5 to $15 \mathrm{ml}$.) collected during the control periods and aliquots of single urine samples collected during the upright and recovery periods were reduced to volumes of less than $1 \mathrm{ml}$., by dialysis overnight through a cellophane bag against 20 per cent Dex$\operatorname{tran}^{6}$ or 25 per cent polyvinyl pyrollidone.? Denaturation or destruction of proteins did not occur provided the contents of the bag were not reduced to dryness.

Following determination of control clearance values during two or three successive ten to twenty-minute intervals with the subject in the recumbent position, clearance values were determined during intervals of ten to twenty-five minutes in the passive upright position with the subject tilted on a tilt table to 40 to $80^{\circ}$ (11 subjects) or half-sitting, half-standing at the end of a bed (8 subjects). Since urine flow fell abruptly and since weakness and hypotension frequently developed within ten to fifteen minutes after standing, only one clearance period could be obtained. Urine collected during the first ten to fifteen minutes of orthostasis was discarded in all but five instances (S. G., H. T., H. Y., M. S., M. L.) in order to permit equilibration. The total interval during the upright position, therefore, varied from twenty to thirty-five minutes. On twelve occasions clearance values were determined over an interval of from fifteen to thirty-five minutes (one or two periods) immediately after the return to the horizontal (recovery). In order to determine whether reduction in clearance values during orthostasis could be attributed to a failure to achieve equilibrium owing to delay in the passage of urine from the glomerulus to the bladder, the urine collected during the first period immediately after return to the horizontal was not discarded. Inclusion of the values for this period in the average values for "recovery" would produce higher values than in the control ("overshoot") if intrarenal delay were an important factor. Glucose $T_{m}$ was determined in six subjects before and during orthostasis in order to assess the distribution of filtrate to functioning tubular tissue. Urine collected during the first ten to fifteen minutes of orthostasis was discarded. Values during recovery (without discard) were measured in five subjects.

\section{RESULTS}

\section{Systemic circulatory adjustments and subjective response}

Within five to thirty minutes after assumption of the upright position evidence of mild to severe circulatory insufficiency (dizziness, sweating, pallor, nausea) appeared in the course of ten of

- Provided through the courtesy of Commercial Solvents Corp., Terre Haute, Indiana.

7 Provided through the courtesy of Schenley Laboratories, Inc., Lawrenceburg, Indiana. twenty studies (E. A., S. G., H. O’N., J. McC., B. W., M. M. No. 1, M. M. No. 2, A. C., E. B., and R. W.). In six of seven subjects in whom arterial pressures were measured (Table I) both systolic and diastolic pressures tended to fall. Although the seventh subject (R.W.), became quite pale and dizzy his arterial pressure was maintained. In all but S. G., who was able to remain upright despite slight weakness and nausea, immediate return to recumbency was necessary in order to prevent syncope. The arterial pressure then returned promptly toward or to control levels and symptoms of circulatory insufficiency disappeared in all but E. B. and R. W., in whom weakness and faintness persisted during the first fifteen minutes of recovery. In the remaining subjects circulatory adjustments during orthostasis were apparently adequate and symptoms of circulatory insufficiency did not develop.

\section{Inulin and sodium p-aminohippurate (PAH) clearances}

The averaged figures for inulin and $\mathrm{PAH}$ clearances during the control period, during orthostasis and during recovery are arranged in Table $I\left(C_{i n}\right.$ and $\mathrm{C}_{\mathrm{PAH}}$ ) according to the control values for the inulin clearances, in descending order. The responses to tilting upright and to standing at the edge of the bed were qualitatively similar, although somewhat greater percentile changes occurred in subjects who were passively tilted. During orthostasis the PAH clearance fell definitely (on the average 41 per cent) in 17 of 20 studies and the inulin clearance decreased similarly (on the average 41 per cent) in 17 instances (Table I, Figure 1). The $\mathrm{PAH}$ clearance was unchanged in three subjects (S. G., R. W., and A. D.) and the inulin clearance in three (S. G., B. W., and A. D.). The inulin and $\mathrm{PAH}$ clearances tended to fall proportionately so that the filtration fraction (F.F., Table I) was not greatly changed. The excessively high values for the filtration fractions in S. G., J. McC., E. B., M. S., and R. W. are probably the result of incomplete extraction of PAH by the diseased kidneys.

On return to the horizontal position the inulin and $\mathrm{PAH}$ clearances promptly returned to or toward control levels in all but two (E. B., and R. W., Table I) of twelve studies. In E. B. and $R$. W., the averaged values for the two recovery 
periods and in S. G. the values during the single "recovery" period (Table I) did not return to control levels owing to continued depression of function and/or intra-renal delay during the first fifteen minutes of recumbency. However, during the last recovery period the clearance values returned to the control level in both E. B. and R. W.
In one subject (H. T.) the clearance values during recovery were somewhat higher than control figures, but in other subjects the control values were not exceeded.

The absence of an "overshoot" in clearance val. ues, as urine flow increased, during recovery strongly supports the view that delay of filtrate

TABLE I $\dagger$

Renal function and protein excretion during orthostasis *

\begin{tabular}{|c|c|c|c|c|c|c|c|c|c|c|c|c|c|c|c|c|}
\hline Sex & $\begin{array}{c}\text { Subject } \\
\text { Diagnosis } \\
\text { Age }\end{array}$ & S.A. & BUN & Procedure & B.P. & T.P. & Het. & $C_{\text {in }}$ & $C_{P A H}$ & FF & $\mathbf{v}$ & $U_{P r}$ & $U_{\mathbf{P r} V}$ & $\begin{array}{l}U_{P_{1}} V_{\text {in }} \\
\end{array}$ & $\mathrm{C}_{\mathrm{Pr}_{\mathrm{r}}}$ & 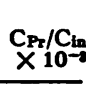 \\
\hline $\mathbf{M}$ & $\begin{array}{c}\text { E. A. } \\
\text { Acute } \\
\text { glom. neph. } \\
48\end{array}$ & $\begin{array}{c}M^{2} \\
2.02\end{array}$ & $\begin{array}{c}\text { mg. \% } \\
19\end{array}$ & $\begin{array}{l}\text { Control } \\
\text { Orthostasis }\end{array}$ & $\begin{array}{c}m m . H_{g} \\
124 / 84 \\
-\end{array}$ & $\begin{array}{l}g m . \\
\% \\
7.03 \\
7.03\end{array}$ & $\begin{array}{l}\% \\
41 \\
41\end{array}$ & $\begin{array}{r}\operatorname{ml} . / \\
\min . \\
131 \\
23\end{array}$ & $\begin{array}{r}\operatorname{ml} . / \\
\min . \\
488 \\
79\end{array}$ & $\begin{array}{l}\% \\
27 \\
29\end{array}$ & $\begin{array}{l}\operatorname{ml} .1 \\
\min . \\
1.7 \\
0.2\end{array}$ & $\begin{array}{c}\text { mg. } \\
\% \\
179 \\
320\end{array}$ & $\begin{array}{c}\text { mg.l } \\
\text { min. } \\
3.1 \\
0.6\end{array}$ & $\begin{array}{c}\mu g_{i} l \\
m i . \\
23 \\
28\end{array}$ & $\begin{array}{l}\text { ml.l } \\
\text { min. } \\
0.05 \\
0.01\end{array}$ & $\begin{array}{l}\% \\
0.3 \\
0.4\end{array}$ \\
\hline M & $\begin{array}{c}\text { S. G. } \\
\text { Chronic } \\
\text { pyeloneph. } \\
25\end{array}$ & & 14 & $\begin{array}{l}\text { Control } \\
\text { Orthostasis } \\
\text { Recovery }\end{array}$ & $\begin{array}{c}120 / 80 \\
106 / 62 \\
-\end{array}$ & $\begin{array}{l}6.35 \\
6.72 \\
6.72\end{array}$ & $\begin{array}{l}51 \\
53 \\
50\end{array}$ & $\begin{array}{l}123 \\
114 \\
110\end{array}$ & $\begin{array}{l}196 \\
191 \\
120\end{array}$ & $\begin{array}{l}63 \\
60 \\
58\end{array}$ & $\begin{array}{l}2.8 \\
1.9 \\
1.3\end{array}$ & $\begin{array}{l}268 \\
211 \\
462\end{array}$ & $\begin{array}{l}7.5 \\
4.0 \\
6.0\end{array}$ & $\begin{array}{l}61 \\
35 \\
55\end{array}$ & $\begin{array}{l}0.12 \\
0.06 \\
0.09\end{array}$ & $\begin{array}{l}1.0 \\
0.5 \\
0.8\end{array}$ \\
\hline $\mathbf{M}$ & $\begin{array}{c}\text { N. H. } \\
\text { Mult. } \\
\text { myeloma } \\
51\end{array}$ & 1.66 & 16 & $\begin{array}{l}\text { Control } \\
\text { Orthostasis }\end{array}$ & $\stackrel{120 / 80}{-}$ & $\begin{array}{l}6.72 \\
6.72\end{array}$ & $\begin{array}{l}41 \\
41\end{array}$ & $\begin{array}{r}118 \\
79\end{array}$ & $\begin{array}{l}660 \\
411\end{array}$ & $\begin{array}{l}18 \\
19\end{array}$ & $\begin{array}{l}1.0 \\
1.4\end{array}$ & $\begin{array}{l}600 \\
336\end{array}$ & $\begin{array}{l}6.0 \\
4.7\end{array}$ & $\begin{array}{l}51 \\
59\end{array}$ & $\begin{array}{l}0.09 \\
0.07\end{array}$ & $\begin{array}{l}0.8 \\
0.9\end{array}$ \\
\hline $\mathbf{F}$ & $\begin{array}{l}\text { L. DiA. } \\
\text { Diss. lupus } \\
\text { erythem. } \\
28\end{array}$ & 1.56 & 7 & $\begin{array}{l}\text { Control } \\
\text { Orthostasis } \\
\text { Recovery }\end{array}$ & $\begin{array}{c}115 / 70 \\
-\end{array}$ & $\begin{array}{l}5.97 \\
5.97 \\
5.97\end{array}$ & $\begin{array}{l}34 \\
36 \\
-\end{array}$ & $\begin{array}{r}105 \\
34 \\
110\end{array}$ & $\begin{array}{l}708 \\
160 \\
505\end{array}$ & $\begin{array}{l}15 \\
21 \\
22\end{array}$ & $\begin{array}{l}3.9 \\
0.8 \\
0.6\end{array}$ & $\begin{array}{l}108 \\
275 \\
667\end{array}$ & $\begin{array}{l}4.2 \\
2.2 \\
4.0\end{array}$ & $\begin{array}{l}40 \\
65 \\
36\end{array}$ & $\begin{array}{l}0.07 \\
0.04 \\
0.07\end{array}$ & $\begin{array}{l}0.7 \\
1.2 \\
0.6\end{array}$ \\
\hline$F$ & $\underset{\substack{\text { Benign } \\
\text { nephroscler. } \\
33}}{\text { W. }}$ & 1.70 & - & $\begin{array}{l}\text { Control } \\
\text { Orthostasis }\end{array}$ & $160 / 120$ & $\begin{array}{l}6.00 \\
6.00\end{array}$ & 39 & $\begin{array}{r}105 \\
63\end{array}$ & $\begin{array}{l}484 \\
266\end{array}$ & $\begin{array}{l}22 \\
24\end{array}$ & $\begin{array}{l}8.5 \\
0.9\end{array}$ & $\begin{array}{r}12 \\
112\end{array}$ & $\begin{array}{l}1.0 \\
1.0\end{array}$ & $\begin{array}{l}10 \\
16\end{array}$ & $\begin{array}{l}0.02 \\
0.02\end{array}$ & $\begin{array}{l}0.2 \\
0.3\end{array}$ \\
\hline $\mathbf{M}$ & $\begin{array}{c}\text { H. O'N. } \\
\text { Acute } \\
\text { glom. neph. } \\
28\end{array}$ & 1.91 & 17 & $\begin{array}{l}\text { Control } \\
\text { Orthostasis }\end{array}$ & $140 / 90$ & $\begin{array}{l}5.97 \\
6.34\end{array}$ & $\begin{array}{l}49 \\
49\end{array}$ & $\begin{array}{r}101 \\
66\end{array}$ & $\begin{array}{l}511 \\
291\end{array}$ & $\begin{array}{l}20 \\
23\end{array}$ & $\begin{array}{l}2.3 \\
0.3\end{array}$ & $\begin{array}{r}209 \\
0\end{array}$ & $\begin{array}{l}4.8 \\
0.0\end{array}$ & $\begin{array}{r}48 \\
0\end{array}$ & $\begin{array}{l}0.08 \\
0.00\end{array}$ & $\begin{array}{l}0.8 \\
0.0\end{array}$ \\
\hline $\mathbf{M}$ & $\begin{array}{c}\text { H. T. } \\
\text { Acute } \\
\text { glom. neph. } \\
26\end{array}$ & 1.69 & 20 & $\begin{array}{l}\text { Control } \\
\text { Orthostasis } \\
\text { Recovery }\end{array}$ & $\begin{array}{c}116 / 110 \\
-\end{array}$ & $\begin{array}{l}5.97 \\
5.97 \\
5.97\end{array}$ & $\begin{array}{l}49 \\
49 \\
-\end{array}$ & $\begin{array}{r}92 \\
38 \\
108\end{array}$ & $\begin{array}{l}604 \\
223 \\
761\end{array}$ & $\begin{array}{l}15 \\
17 \\
14\end{array}$ & $\begin{array}{l}4.9 \\
1.7 \\
1.8\end{array}$ & $\begin{array}{l}110 \\
194 \\
239\end{array}$ & $\begin{array}{l}5.4 \\
3.3 \\
4.3\end{array}$ & $\begin{array}{l}59 \\
87 \\
40\end{array}$ & $\begin{array}{l}0.09 \\
0.06 \\
0.07\end{array}$ & $\begin{array}{l}1.0 \\
1.6 \\
0.7\end{array}$ \\
\hline $\mathbf{F}$ & $\begin{array}{l}\text { M. C. } \\
\text { Diss. lupus } \\
\text { erythem. } \\
20\end{array}$ & 1.56 & 23 & $\begin{array}{l}\text { Control } \\
\text { Orthostasis }\end{array}$ & $110 / 70$ & $\begin{array}{l}5.41 \\
5.41\end{array}$ & $\begin{array}{l}32 \\
31\end{array}$ & $\begin{array}{l}63 \\
33\end{array}$ & $\begin{array}{l}482 \\
267\end{array}$ & $\begin{array}{l}13 \\
12\end{array}$ & $\begin{array}{l}5.5 \\
3.2\end{array}$ & $\begin{array}{l}40 \\
25\end{array}$ & $\begin{array}{l}2.2 \\
0.8\end{array}$ & $\begin{array}{l}36 \\
23\end{array}$ & $\begin{array}{l}0.04 \\
0.01\end{array}$ & $\begin{array}{l}0.7 \\
0.4\end{array}$ \\
\hline
\end{tabular}

* All control clearance values are averages of two or more determinations. All other clearance values represent single determinations except in instances marked with an asterisk, which are averages of two determinations. $\dagger$ Abbreviations are as follows:

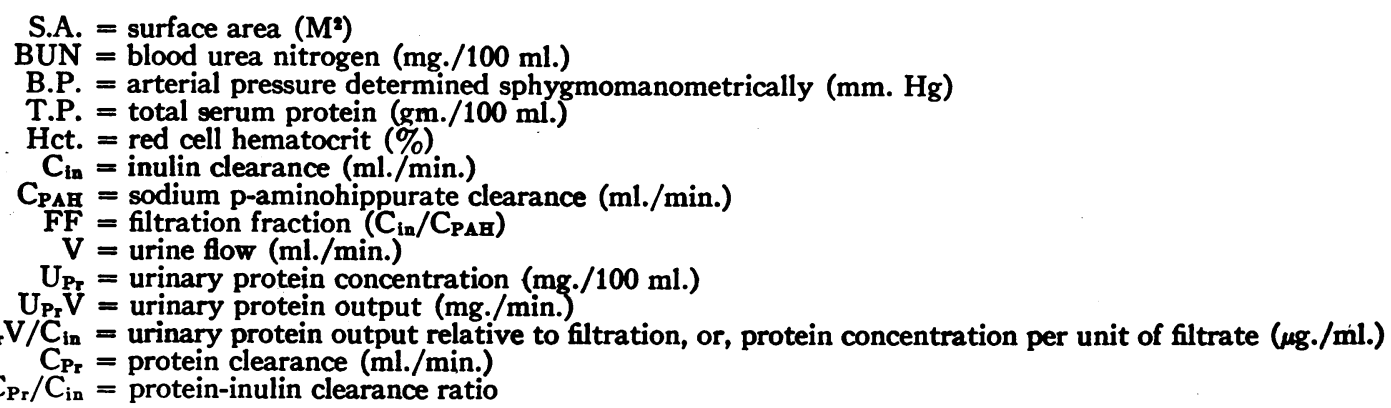


TABLE I-Continued

\begin{tabular}{|c|c|c|c|c|c|c|c|c|c|c|c|c|c|c|c|c|}
\hline Sex & $\begin{array}{c}\text { Subject } \\
\text { Diagnocis } \\
\text { Age }\end{array}$ & S.A. & BUN & Procedure & B.P. & T.P. & Hct. & $C_{\text {in }}$ & $\mathrm{C}_{\mathrm{PAB}}$ & FF & v & $U_{P_{r}}$ & URtV & $\mathrm{UPR}_{\mathrm{Pin}} \mathrm{VI}$ & $C_{P r}$ & 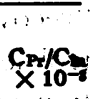 \\
\hline $\mathbf{M}$ & $\underset{\substack{\text { Benign } \\
\text { nephroscler. } \\
54}}{\text { McC. }}$ & $\begin{array}{c}M^{2} \\
1.74\end{array}$ & $\begin{array}{c}m g . \% \\
24\end{array}$ & $\begin{array}{l}\text { Control } \\
\text { Orthostasis }\end{array}$ & $\begin{array}{c}m m . H g \\
250 / 150 \\
200 / 140\end{array}$ & $\begin{array}{l}8 m . \\
\% .34 \\
6.72\end{array}$ & $\begin{array}{l}\% \\
44 \\
46\end{array}$ & $\begin{array}{r}\text { ml!l } \\
\text { min. } \\
59 \\
37\end{array}$ & $\begin{array}{c}m ! \cdot l \\
m i n . \\
126 \\
98\end{array}$ & $\begin{array}{l}\% \\
47 \\
38\end{array}$ & $\begin{array}{l}\text { ml.l } \\
\text { min. } \\
4.2 \\
0.8\end{array}$ & $\begin{array}{c}m g . \\
\% \\
67 \\
200\end{array}$ & $\begin{aligned} \operatorname{mg}_{\text {gin. }} \\
2.8 \\
1.6\end{aligned}$ & $\begin{array}{c}\mu_{8} ! \\
47 \\
43\end{array}$ & $\begin{array}{l}\text { ml.1. } \\
\text { min. } \\
0.04 \\
1.02\end{array}$ & $\begin{array}{l}\% \\
0.7 \\
0.6\end{array}$ \\
\hline $\mathbf{M}$ & $\begin{array}{c}\text { W. M. } \\
\text { Chronic } \\
\text { glom. neph. } \\
\mathbf{4 4}\end{array}$ & 1.86 & 22 & $\begin{array}{l}\text { Control } \\
\text { Orthostasis } \\
\text { Recovery* }\end{array}$ & $\begin{array}{l}160 / 100 \\
138 / 96 \\
132 / 70\end{array}$ & $\begin{array}{l}4.01 \\
4.48 \\
4.48\end{array}$ & $\begin{array}{l}30 \\
33 \\
32\end{array}$ & $\begin{array}{l}57 \\
40 \\
54\end{array}$ & $\begin{array}{l}467 \\
312 \\
470\end{array}$ & $\begin{array}{l}12 \\
13 \\
11\end{array}$ & $\begin{array}{l}5.0 \\
1.9 \\
2.5\end{array}$ & $\begin{array}{l}140 \\
363 \\
196\end{array}$ & $\begin{array}{l}7.0 \\
6.9 \\
4.9\end{array}$ & $\begin{array}{r}120 \\
170 \\
90\end{array}$ & $\begin{array}{l}0.18 \\
0.15 \\
0.11\end{array}$ & $\begin{array}{l}3.2 \\
3.8 \\
2.0\end{array}$ \\
\hline F & $\begin{array}{c}\text { B. W. } \\
\text { Acute } \\
\text { glom. neph. } \\
15\end{array}$ & 1.83 & 14 & $\begin{array}{l}\text { Control } \\
\text { Orthostasis } \\
\text { Recovery }\end{array}$ & $\begin{array}{r}106 / 66 \\
70 / 30 \\
110 / 70\end{array}$ & $\begin{array}{l}6.72 \\
7.09 \\
6.90\end{array}$ & $\frac{40}{39}$ & $\begin{array}{l}55 \\
48 \\
54\end{array}$ & $\begin{array}{l}432 \\
313 \\
360\end{array}$ & $\begin{array}{l}13 \\
15 \\
15\end{array}$ & $\begin{array}{l}4.7 \\
2.4 \\
2.4\end{array}$ & $\begin{array}{l}53 \\
53 \\
63\end{array}$ & $\begin{array}{l}2.5 \\
1.6 \\
1.5\end{array}$ & $\begin{array}{l}50 \\
33 \\
30\end{array}$ & $\begin{array}{l}0.04 \\
0.02 \\
0.02\end{array}$ & $\begin{array}{l}0.7 \\
0.4 \\
0.4\end{array}$ \\
\hline $\mathbf{M}$ & $\begin{array}{c}\text { H. Y. } \\
\text { Benign } \\
\text { nephroscler. } \\
26\end{array}$ & 1.78 & 15 & $\begin{array}{l}\text { Control } \\
\text { Orthostasis } \\
\text { Recovery }\end{array}$ & $\begin{array}{c}180 / 100 \\
=\end{array}$ & $\begin{array}{l}5.22 \\
5.60 \\
4.85\end{array}$ & $\begin{array}{l}48 \\
50 \\
48\end{array}$ & $\begin{array}{l}48 \\
37 \\
54\end{array}$ & $\begin{array}{l}169 \\
114 \\
170\end{array}$ & $\begin{array}{l}28 \\
32 \\
32\end{array}$ & $\begin{array}{l}1.9 \\
0.7 \\
0.9\end{array}$ & $\begin{array}{r}479 \\
972 \\
1266\end{array}$ & $\begin{array}{r}9.1 \\
6.8 \\
11.4\end{array}$ & $\begin{array}{l}190 \\
180 \\
210\end{array}$ & $\begin{array}{l}0.17 \\
0.13 \\
0.23\end{array}$ & $\begin{array}{l}3.5 \\
3.5 \\
4.3\end{array}$ \\
\hline F & $\begin{array}{l}\text { M. M. No. } 1 \\
\text { Chronic } \\
\text { glom. neph. } \\
22\end{array}$ & 1.60 & 31 & $\begin{array}{l}\text { Control } \\
\text { Orthostasis } \\
\text { Recovery }\end{array}$ & $\begin{array}{l}194 / 100 \\
120 / 80 \\
192 / 120\end{array}$ & $\begin{array}{l}4.85 \\
4.85 \\
4.66\end{array}$ & $\begin{array}{l}37 \\
37 \\
35\end{array}$ & $\begin{array}{l}31 \\
12 \\
19\end{array}$ & $\begin{array}{r}193 \\
74 \\
126\end{array}$ & $\begin{array}{l}16 \\
16 \\
15\end{array}$ & $\begin{array}{l}1.9 \\
0.4 \\
0.6\end{array}$ & $\begin{array}{l}484 \\
626 \\
850\end{array}$ & $\begin{array}{l}9.2 \\
2.5 \\
5.1\end{array}$ & $\begin{array}{l}300 \\
210 \\
270\end{array}$ & $\begin{array}{l}0.19 \\
0.05 \\
0.11\end{array}$ & $\begin{array}{l}6.1 \\
4.3 \\
5.7\end{array}$ \\
\hline $\mathbf{M}$ & $\underset{\substack{\text { A. C. C. } \\
\text { glom. neph. } \\
\mathbf{3 7}}}{\stackrel{\text {. }}{\text { Ant }}}$ & 1.78 & 41 & $\begin{array}{l}\text { Control } \\
\text { Orthostasis } \\
\text { Recovery }\end{array}$ & $\begin{array}{l}200 / 120 \\
180 / 120 \\
198 / 120\end{array}$ & $\begin{array}{l}8.21 \\
8.58 \\
7.84\end{array}$ & $\begin{array}{l}37 \\
38 \\
38\end{array}$ & $\begin{array}{l}26 \\
17 \\
26\end{array}$ & $\begin{array}{r}102 \\
64 \\
99\end{array}$ & $\begin{array}{l}26 \\
27 \\
27\end{array}$ & $\begin{array}{l}6.9 \\
2.8 \\
4.6\end{array}$ & $\begin{array}{r}144 \\
61 \\
143\end{array}$ & $\begin{array}{l}9.9 \\
1.7 \\
6.8\end{array}$ & $\begin{array}{l}370 \\
120 \\
260\end{array}$ & $\begin{array}{l}0.12 \\
0.02 \\
0.09\end{array}$ & $\begin{array}{l}4.0 \\
1.0 \\
3.0\end{array}$ \\
\hline F & $\begin{array}{c}\text { M. M. No. } 2 \\
\text { Chronic } \\
\text { glom. neph. } \\
22\end{array}$ & 1.60 & 31 & $\begin{array}{l}\text { Control } \\
\text { Orthostasis } \\
\text { Recovery* }\end{array}$ & $\begin{array}{c}160 / 100 \\
90 / 50 \\
140 / 90\end{array}$ & $\begin{array}{l}4.85 \\
4.85 \\
4.66\end{array}$ & $\begin{array}{l}36 \\
36 \\
35\end{array}$ & $\begin{array}{r}22 \\
2 \\
20\end{array}$ & $\begin{array}{r}185 \\
15 \\
188\end{array}$ & $\begin{array}{l}12 \\
12 \\
11\end{array}$ & $\begin{array}{l}2.6 \\
0.1 \\
0.8\end{array}$ & $\begin{array}{r}238 \\
60 \\
650\end{array}$ & $\begin{array}{l}6.2 \\
0.6 \\
5.2\end{array}$ & $\begin{array}{l}280 \\
300 \\
260\end{array}$ & $\begin{array}{l}0.13 \\
0.01 \\
0.11\end{array}$ & $\begin{array}{l}5.9 \\
5.0 \\
5.1\end{array}$ \\
\hline $\mathbf{M}$ & $\underset{\substack{\text { E. B. } \\
\text { Malig. } \\
\text { nephroscler. }}}{\text {. }}$ & 2.01 & 39 & $\begin{array}{l}\text { Control } \\
\text { Orthostasis } \\
\text { Recovery* }\end{array}$ & $\begin{array}{c}200 / 110 \\
=\end{array}$ & $\frac{6.72}{6.72}$ & $\frac{30}{29}$ & $\begin{array}{l}20 \\
14 \\
14\end{array}$ & $\begin{array}{l}28 \\
21 \\
24\end{array}$ & $\begin{array}{l}71 \\
67 \\
58\end{array}$ & $\begin{array}{l}3.3 \\
1.5 \\
1.5\end{array}$ & $\begin{array}{r}94 \\
133 \\
86\end{array}$ & $\begin{array}{l}3.1 \\
2.0 \\
1.3\end{array}$ & $\begin{array}{r}160 \\
140 \\
90\end{array}$ & $\begin{array}{l}0.05 \\
0.03 \\
0.02\end{array}$ & $\begin{array}{l}2.4 \\
2.1 \\
1.5\end{array}$ \\
\hline M & 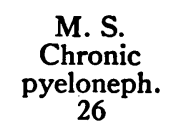 & 2.06 & 41 & $\begin{array}{l}\text { Control } \\
\text { Orthostasis }\end{array}$ & $210 / 130$ & $\begin{array}{l}5.97 \\
6.34\end{array}$ & $\begin{array}{l}27 \\
28\end{array}$ & $\begin{array}{l}9 \\
4\end{array}$ & $\begin{array}{l}19 \\
10\end{array}$ & $\begin{array}{l}47 \\
40\end{array}$ & $\begin{array}{l}4.1 \\
1.6\end{array}$ & $\begin{array}{l}200 \\
256\end{array}$ & $\begin{array}{l}8.2 \\
4.1\end{array}$ & $\begin{array}{r}910 \\
1020\end{array}$ & $\begin{array}{l}0.14 \\
0.06\end{array}$ & $\begin{array}{l}16.0 \\
15.0\end{array}$ \\
\hline $\mathbf{M}$ & $\begin{array}{c}\text { R. W. } \\
\text { Chronic } \\
\text { glom. neph. } \\
58\end{array}$ & 1.70 & 93 & $\begin{array}{l}\text { Control } \\
\text { Orthostasis } \\
\text { Recovery* }\end{array}$ & $\begin{array}{l}180 / 100 \\
180 / 92 \\
190 / 90\end{array}$ & $\begin{array}{l}7.84 \\
7.84 \\
7.84\end{array}$ & $\begin{array}{l}20 \\
20 \\
20\end{array}$ & $\begin{array}{l}8 \\
6 \\
6\end{array}$ & $\begin{array}{l}13 \\
12 \\
11\end{array}$ & $\begin{array}{l}62 \\
50 \\
54\end{array}$ & $\begin{array}{l}3.7 \\
2.7 \\
2.4\end{array}$ & $\begin{array}{r}108 \\
74 \\
105\end{array}$ & $\begin{array}{l}4.0 \\
2.0 \\
2.5\end{array}$ & $\begin{array}{l}490 \\
340 \\
420\end{array}$ & $\begin{array}{l}0.05 \\
0.03 \\
0.03\end{array}$ & $\begin{array}{l}6.0 \\
4.0 \\
4.0\end{array}$ \\
\hline$F$ & $\begin{array}{c}\text { M. L. } \\
\text { Chronic } \\
\text { glom. neph. } \\
33\end{array}$ & 1.68 & 105 & $\begin{array}{l}\text { Control } \\
\text { Orthostasis } \\
\text { Recovery }\end{array}$ & $\begin{array}{c}210 / 110 \\
=\end{array}$ & $\begin{array}{l}5.22 \\
5.97\end{array}$ & $\begin{array}{l}19 \\
19\end{array}$ & $\begin{array}{l}5 \\
2 \\
3\end{array}$ & $\begin{array}{l}69 \\
40 \\
62\end{array}$ & $\begin{array}{l}8 \\
6 \\
5\end{array}$ & $\begin{array}{l}1.8 \\
0.8 \\
1.1\end{array}$ & $\begin{array}{r}183 \\
225 \\
73\end{array}$ & $\begin{array}{l}3.3 \\
1.8 \\
0.8\end{array}$ & $\begin{array}{l}630 \\
780 \\
260\end{array}$ & $\begin{array}{l}0.06 \\
0.03 \\
0.01\end{array}$ & $\begin{array}{r}12.0 \\
13.0 \\
3.0\end{array}$ \\
\hline $\mathbf{M}$ & $\underset{\substack{\text { A. Dronic } \\
\text { glom.neph. }}}{\stackrel{\text {. }}{35}}$ & 1.49 & 126 & $\begin{array}{l}\text { Control } \\
\text { Orthostasis }\end{array}$ & $110 / 80$ & $\begin{array}{l}6.71 \\
6.34\end{array}$ & $\begin{array}{l}27 \\
25\end{array}$ & $\begin{array}{l}4 \\
4\end{array}$ & $\begin{array}{l}30 \\
30\end{array}$ & $\begin{array}{l}14 \\
14\end{array}$ & $\begin{array}{l}1.2 \\
1.1\end{array}$ & $\begin{array}{r}217 \\
64\end{array}$ & $\begin{array}{l}2.6 \\
0.7\end{array}$ & $\begin{array}{l}60 \\
17\end{array}$ & $\begin{array}{l}0.04 \\
0.01\end{array}$ & $\begin{array}{l}9.4 \\
2.4\end{array}$ \\
\hline
\end{tabular}

within the tubules during orthostasis did not contribute importantly to the changes observed during orthostasis. These changes may be ascribed to decrements in renal blood flow and glomerular filtration. Since impaired renal extraction of $\mathrm{PAH}$ is characteristic of chronic renal disease (9), a further acute reduction in extraction might ac- count for the fall in PAH clearance. However, the degree of fall in PAH clearance, its association with a similar decrease in inulin clearance and its reversibility support the view that diminished PAH excretion under these circumstances was the result of reduced delivery of PAH by the blood to tubular cells. 


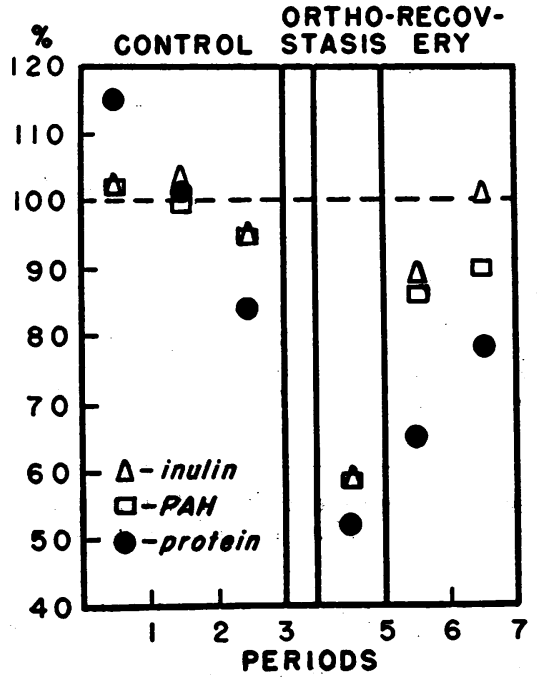

Fig. 1. The Effect of Orthostasis on the Clearances of INULIN aNd PAH aNd ON URINARy Protein EXCRETION

The values for inulin and PAH clearances and for protein output have been averaged for each period and expressed as the percentage of the pooled and averaged control figures. All data presented here represent complete experiments in twelve subjects (Table I) except for the second recovery period which represent values of four subjects (W. M., M. M. No. 2, E. B., and R. W.) only. All values fell sharply and proportionately during orthostasis and returned toward control levels during recovery.

\section{Tubular transfer maximum (glucose $T_{m}$ )}

The maximal rate of tubular reabsorption of glucose was measured in six subjects (Table II). In all inulin clearance and glucose $T_{m}$ fell to approximately the same extent, so that the inulin clearance-glucose $T_{m}$ ratio $\left(C_{m} / T_{m g}\right.$, Figure 2$)$ remained relatively constant. During recovery glucose $T_{m}$ returned to control levels in four of the five instances studied.

Whether complete saturation of all tubules had been effected in these subjects could not be determined with certainty. Even at high plasma glucose levels saturation of the tubules may be difficult to achieve in advanced renal disease because filtration may be so greatly reduced in many nephrons. When the load imposed upon the tubules is reduced by a further fall in filtration, such as that which occurs during orthostasis, glucose $T_{m}$ may appear to decrease simply because the load has decreased in proportion to filtration. The filtered load of glucose fell below the control value for the maximal rate of reabsorption in $\mathrm{J} . \mathrm{McC}$. during orthostasis (load/ $\mathrm{T}_{\mathrm{mg}}$ [Table II] ratio less than one). In $W . M$. the load $/ T_{m g}$ ratio fell to 1.04 , but in E. B., B. W., and A. C. the load remained much higher than the control value for $T_{m g}$ In view of the fall in $T_{m g}$ even when the load was sustained at a high level and the proportionate reduction in $T_{m g}$ and inulin clearance even when the calculated load decreased below the control rate of glucose reabsorption it seems most likely that the decrement in $T_{m g}$ may be ascribed to cessation of filtration in a proportion of the glomeruli. Since an excess of glucose was not excreted during recovery, it may be inferred that delay within the urinary passages did not contribute significantly to the fall in glucose $T_{m}$ during orthostasis. The changes noted in $\mathrm{C}_{\mathrm{in}} / \mathrm{T}_{\mathrm{mg}}$ ratio during recovery cannot be explained but they are possibly attributable to inclusion of urine collected during the period of equilibration immediately after return to the horizontal in order to detect "overshoot."

\section{Protein excretion}

The excretion of protein in the urine decreased sharply in 18 instances, and remained unchanged in two (J. W. and W. M.) ( $U_{p r} V$, Table $\left.I\right)$. The failure to detect a change in proteinuria in $\mathrm{J}$. W. may have been due to technical errors since the control urinary protein concentration $(12 \mathrm{mg}$. per

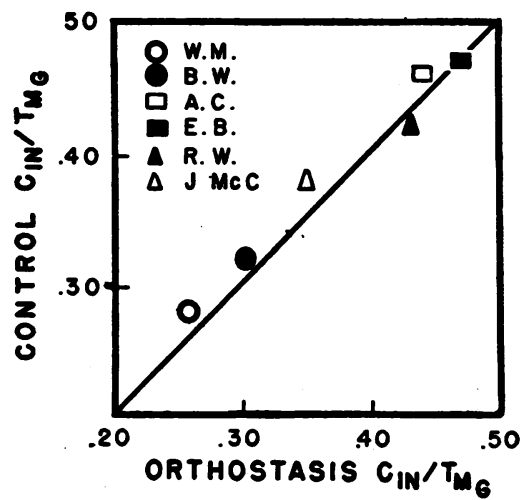

Fig. 2. Distribution of Glomerular Filtrate to Functioning Tubular Tissue

The filtration rate $\left(C_{1 n}\right)$ relative to maximal glucose reabsorptive capacity $\left(T_{m_{8}}\right)$ during the control period is plotted against this ratio during orthostasis. In all subjects filtration and $T_{m g}$ fell proportionately, presumably indicating that filtration diminished entirely as a result of cessation of glomerular activity. 
TABLE II

Effect of orthostasis upon maximal glucose reabsorptive capacity $\left(T_{m_{g}}\right)$ *

\begin{tabular}{|c|c|c|c|c|c|c|c|c|c|c|c|c|}
\hline \multirow[b]{2}{*}{ Subject } & \multicolumn{4}{|c|}{ Control } & \multicolumn{4}{|c|}{ Orthostasis } & \multicolumn{4}{|c|}{ Recovery } \\
\hline & $\begin{array}{l}\text { Plasma } \\
\text { glucose }\end{array}$ & $\frac{\text { Loadt }}{T_{m g}}$ & $\underset{T_{m}}{\text { Glucose }}$ & $\mathrm{C}_{\mathrm{in}} / \mathrm{T}_{\mathrm{me}}$ & $\begin{array}{l}\text { Plasma } \\
\text { glucose }\end{array}$ & $\frac{\text { Loadt }}{T_{\text {mg }}}$ & $\underset{T_{m}}{\text { Glucose }}$ & $\mathrm{C}_{\text {in }} / \mathrm{T}_{\mathrm{me}} \neq$ & $\begin{array}{l}\text { Plasma } \\
\text { glucose }\end{array}$ & $\frac{\text { Loadt }}{T_{m E}}$ & $\underset{T_{m}}{\text { Glucose }}$ & $\mathrm{C}_{\text {in }} / \mathrm{T}_{\text {me }} \neq$ \\
\hline $\begin{array}{l}\text { J. McC. } \\
\text { W. M. } \\
\text { B. W. } \\
\text { A. C. } \\
\text { E. B. } \\
\text { R. W. }\end{array}$ & $\begin{array}{c}\text { mg. } \% \\
400 \\
487 \\
632 \\
601 \\
378 \\
609\end{array}$ & $\begin{array}{l}1.5 \\
1.3 \\
2.0 \\
2.8 \\
1.8 \\
2.6\end{array}$ & $\begin{array}{c}\text { mg./min. } \\
158 \\
206 \\
173 \\
59 \\
43 \\
19\end{array}$ & $\begin{array}{l}0.38 \\
0.28 \\
0.32 \\
0.46 \\
0.47 \\
0.42\end{array}$ & $\begin{array}{c}\text { mg. \% } \\
335 \\
525 \\
574 \\
614 \\
395 \\
650\end{array}$ & $\begin{array}{l}0.8 \\
1.04 \\
1.6 \\
1.8 \\
1.3 \\
2.1\end{array}$ & $\begin{array}{c}\text { mg./min. } \\
102 \\
151 \\
156 \\
39 \\
30 \\
14\end{array}$ & $\begin{array}{l}0.36 \\
0.27 \\
0.31 \\
0.44 \\
0.47 \\
0.43\end{array}$ & $\begin{array}{c}\text { mg. \% } \\
534 \\
560 \\
620 \\
417 \\
698\end{array}$ & $\begin{array}{l}-\overline{1.4} \\
1.7 \\
2.8 \\
1.4 \\
2.2\end{array}$ & $\begin{array}{c}\text { mg./min. } \\
206^{*} \\
188 \\
65 \\
38^{*} \\
11^{*}\end{array}$ & $\begin{array}{l}0.26 \\
0.29 \\
0.40 \\
0.37 \\
0.55\end{array}$ \\
\hline
\end{tabular}

* All control values are averages of three determinations. All other values represent single determinations except those instances marked with an asterisk $\left({ }^{*}\right)$, which are averages of two periods. Values for urine flow, $C_{\text {in }}$ and $C_{P A B}$ obtained simultaneously in these patients are presented in Table $I$.

Abbreviations as in Table I except as follows:

$\dagger$ Load $/ \mathrm{T}_{\mathrm{mg}}=$ glucose filtered (arterial glucose concentration $(\mathrm{mg} . / \mathrm{ml}$.$) \times inulin clearance (\mathrm{ml} . / \mathrm{min}$.)) relative to maximal reabsorptive capacity $\left(\mathrm{T}_{\mathrm{m \varepsilon}}\right)$.

$\ddagger \mathrm{C}_{\mathrm{in}} / \mathrm{T}_{\mathrm{mg}}=$ glomerular filtration rate relative to maximal tubular reabsorptive capacity (ml./mg.), using the value for $T_{m \varepsilon}$ obtained during the control periods.

cent) was too low for accurate measurement. The excretion of protein varied widely from subject to subject (1.0 to $9.9 \mathrm{mg}$. per min.) and showed a tendency to fall slightly during the control periods. Nevertheless, the fall in protein excretion during orthostasis is highly significant $(t=2.81$, $\mathrm{p}<.01$ ) when compared with spontaneous variations in proteinuria observed over a similar time interval in a group of eight proteinuric subjects studied during recumbency only.

On returning to the horizontal position (12 studies) protein output tended to return toward, or to, control levels in all but five subjects (E. B., R. W., W. M., B. W., and M. L.) in whom the reduction in protein excretion persisted. The control value was exceeded on only one occasion (H. Y.) during recovery.

On the average, the excretion of urinary protein decreased 49 per cent during orthostasis, a value corresponding closely to the average changes in inulin and PAH clearances (Figure 1). The return of protein excretion to control levels was somewhat less rapid than the recovery in inulin and PAH clearances. Since plasma protein concentration remained relatively constant in most subjects total protein clearances showed directional and percentile changes similar to those for total protein excretion (Table I). However, in a few subjects the plasma protein concentrations increased slightly owing to hemoconcentration (parallel increments in the hematocrit were usually observed) during gravitational pooling of blood in the lower extremities; hence, protein clearances tended to change less than protein output. Protein excretion fell relatively more than urine flow in eight instances (S. G., N. H., H. O'N., M. C., A. C., M. M. No. 2, R. W., and A. D.), and urinary protein concentration decreased. In $\mathrm{B}$. W. the urinary concentration of protein did not change but in the remaining eleven studies it increased.

In general the decrease in protein excretion during orthostasis was roughly proportional to the fall in filtration, but disproportionate reductions in proteinuria did occur. Protein excretion and clearance fell less in five subjects (L. D'A., S. W., H. T., W. M., and M. L.) and more in eight (S. G., H. O’N., M. C., B. W., M. M. No. 1, A. C., R. W., and A. D.) than the inulin clearance (Table I). In J. W. and W. M. the inulin clearance fell 40 and 30 per cent, respectively, without changes in protein output, whereas in A. D. protein excretion fell significantly without an apparent change in filtration. However, in A. D. the inulin clearance was so greatly reduced by disease $(4 \mathrm{ml}$. per min.) that a further significant reduction may have been too small to detect.

Satisfactory patterns of plasma (or serum) and urinary proteins by paper electrophoresis were obtained in seven subjects before and during orthostasis. Owing to difficulty in photographic reproduction (lack of contrast) the results of four experiments only are illustrated in Figure 3. 


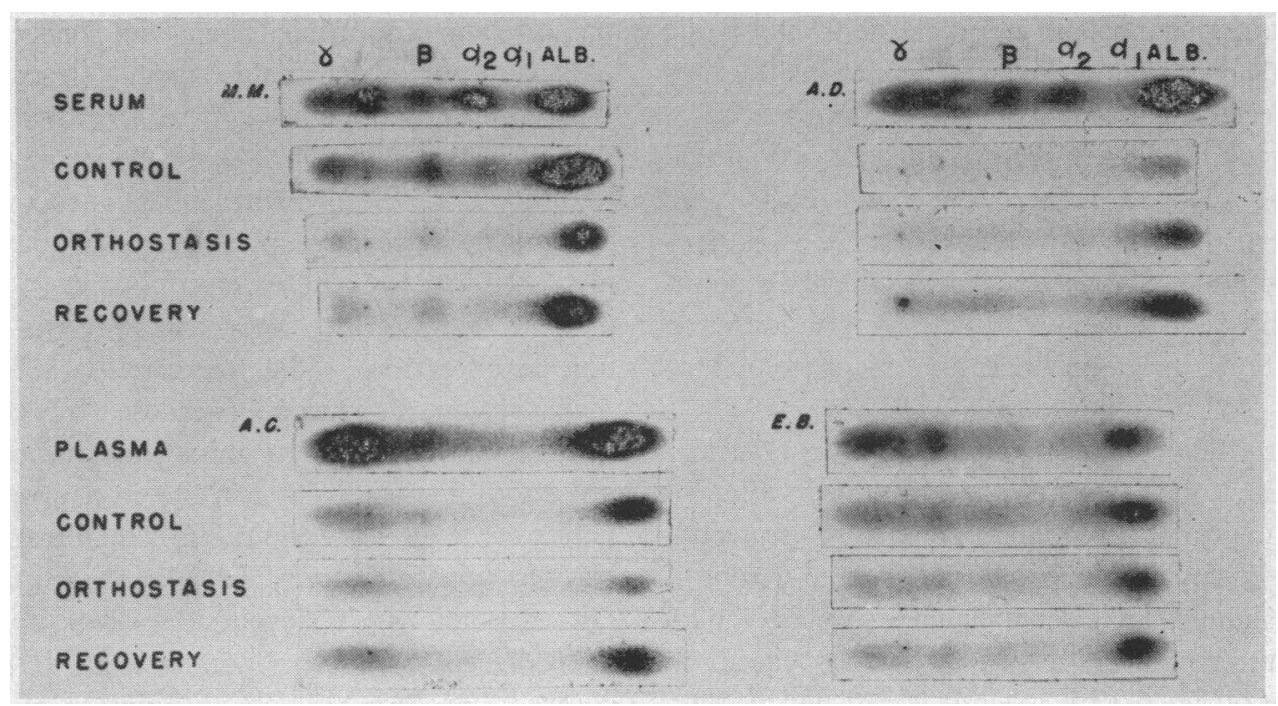

Fig. 3. Paper Electrophoretic Patterns of Urinary Proteins During Control Orthostasis AND Recovery

No change from the control pattern was detectable in the urinary protein mixture during or after orthostasis, other than that attributable to dilution of all fractions, as a result of "washing out" the bladder. Hence, all protein fractions appeared to participate in the reduction of protein excretion; none was selectively affected to a demonstrably greater or less exțent than the others.

\section{DISCUSSION}

In normal man, the assumption of the upright position sets in train widespread vasoconstriction in which the renal vasculature participates (18, 19). The present study demonstrates the persistence of such an intra-renal vasoconstrictive response in severe acute or chronic disease of the kidney. Undoubtedly, the magnitude of the observed response was exaggerated to some extent by vasomotor instability and inadequate maintenance of arterial pressure in patients who were ill and bedfast. In almost every instance, effective renal plasma flow decreased relatively more than the mean brachial arterial pressure shortly after tilting or standing upright. Glomerular filtration usually fell in proportion to renal plasma flow. Urine flow declined and the urinary protein concentration tended to increase, thus accounting for the clinical observation of "postural proteinuria" in the course of chronic renal disease. However, total protein excretion usually decreased to about the same extent as filtration. Hence, intra-renal vasoconstriction appeared to affect protein output by reducing protein filtration rather than by changing glomerular capillary permeability.

Since the glomerular filtration rate and the maximal tubular reabsorptive capacity for glucose $\left(T_{m g}\right)$ appeared to decrease proportionately, the fall in filtration may be ascribed to a diminution in the area of the filtering surface, presumably as a result of cessation of function in a fraction of the nephrons. A similar fall in filtration and $\mathrm{T}_{\mathrm{mg}}$ has also been observed in normal subjects during orthostasis (20). The glomerular injury in the diseased kidney is notably diverse (21), ranging from complete obliteration of some units to absence of demonstrable anatomical alteration in others. Hence, the fact that protein excretion, filtration rate, and glucose $T_{m}$ all appeared to decrease to more or less the same extent in this study strongly suggests that vasoconstriction within the diseased kidney during orthostasis does not affect any category of glomeruli preferentially. The selective elimination of the less permeable glomeruli from function would be expected to reduce albumin excretion more than globulin excretion, if it may be assumed that glomerular permeability under these circumstances is primarily a function of molecular size. Albumin excretion would then decrease more and globulin excretion less than total protein output. 
On the other hand, selective elimination of the more permeable glomeruli would have the opposite effect of increasing albumin output relative to globulin. However, paper elctrophoresis failed to disclose any detectable change in urinary protein pattern during or after orthostasis, thus providing further evidence that the glomerular population which remains in function is a random sample of the total in operation prior to orthostasis.

This study was not designed to test critically the question of the relative contributions of glomerular and tubular derangements in the mechanism of proteinuria. It has been assumed throughout that changes in glomerular permeability play a predominant role, but the inferences based on this assumption, which are set forth here, apply with equal force if a defect in tubular protein reabsorption is of importance. The variability in the character of protein loss from patient to patient and the lack of a clear-cut pattern of predictable change throughout the course of renal disease noted in these data (Table I and Figure 3) suggest that factors other than the shape and dimensions of the plasma protein molecules may determine to some extent their escape into the urine. Thus, in one subject in terminal uremia (A. D., Figure 3 ) the urinary protein was almost entirely albumin, whereas in another with much less functional impairment (M. M.) the urinary protein pattern closely resembled that of the patient's plasma and contained a large amount of globulin. On the whole, however, total protein loss was correlated with the available filtering surface (Table I), decreasing as filtration declined with advance in the disease process. Moreover, protein escape apparently decreased less rapidly than filtration during the course of disease (Table I). As a result, the protein output-inulin clearance ratio tended to be high when the kidney was severely damaged, conforming with the widely-accepted view that glomerular permeability tends to increase with progression of disease $(3,6,7,8$, $22,23)$.

\section{SUMMARY}

An investigation of the effect of the renal hemodynamic adjustments during orthostasis on protein excretion was made in nineteen patients with acute and chronic renal disease, Glomerular fil- tration rate (inulin clearance) and effective renal plasma flow (p-aminohippurate clearance) decreased almost immediately after assumption of the upright position in nearly every instance, apparently as a result of intra-renal vasoconstriction. Although the urinary protein concentration often increased under these circumstances the total protein output fell to approximately the same extent as filtration. The maximal rate of tubular reabsorption of glucose (glucose $\mathrm{T}_{\mathrm{m}}$ ) decreased with filtration in six determinations, indicating that cessation of glomerular activity in a proportion of nephrons accounted for the fall in filtration. Filtration, protein output, and glucose $T_{m}$ tended to fall proportionately despite the diversity inherent in a diseased nephron population. It was concluded, therefore, that the population of nephrons remaining in operation during orthostasis did not differ substantially from the total. All constituents of the urinary protein mixture seemed equally affected since no obvious changes in urinary protein patterns obtained by paper electrophoresis were observed during or after orthostasis. This finding was interpreted as further evidence of a diffuse and relatively uniform vasoconstrictive response that did not significantly alter glomerular capillary permeability.

\section{ACKNOWLEDGMENTS}

We wish to express our gratitude to Mrs. Katherine T. Baker, Mrs. Joan H. Banfield, Miss Barbara Jones, Miss Phyllis Kallenberg, and Mrs. Lottie F. Klayman for their efficient and valuable technical assistance.

\section{REFERENCES}

1. Lathem, W., Roof, B. S., and Nickel, J. F., The effect of the upright position on protein excretion and renal function in renal disease. J. Clin. Invest., 1953, 32, 582.

2. Bradley, S. E., and Tyson, C. J., The "nephrotic syndrome." New England J. Med., 1948, 238, 223 and 260 .

3. Barnett, H. L., Forman, C. W., and Lauson, H. D., The nephrotic syndrome in children. Advances in Pediat., 1952, 5, 53.

4. Rather, L. J., Filtration, resorption, and excretion of protein by the kidney. Medicine, 1952, 31, 357.

5. Berglund, H., Medes, G., Huber, G. C., Longcope, W. T., and Richards, A. N., eds., The Kidney in Health and Disease in Contributions by Eminent Authorities. Philadelphia, Lea and Febiger, 1935.

6. Bing, J., Studies on proteinuria. Acta med. Scandinav., (Suppl.), 1936, 76, 1. 
7. Hiller, A., McIntosh, J. F., and Van Slyke, D. D., The excretion of albumin and globulin in nephritis. J. Clin. Invest., 1927, 4, 235.

8. Blackman, S. S., Jr., Goodwin, W. E., and Buell, M. V., On the relation between the concentration of total protein and of globulin in the urine and the pathogenesis of certain renal lesions in Bright's disease. Bull. Johns Hopkins Hosp., 1941, 69, 397.

9. Bradley, S. E., Bradley, G. P., Tyson, C. J., Curry, J. J., and Blake, W. D., Renal function in renal diseases. Am. J. Med, 1950, 9, 766.

10. Derow, H. A., The diagnostic value of serial measurements of albuminuria in ambulatory patients. New England J. Med., 1942, 227, 827.

11. White, H. L., and Rolf, D., Effects of exercise and of some other influences on the renal circulation in man. Am. J. Physiol., 1948, 152, 505.

12. Starr, I., Jr., The production of albuminuria by renal vasoconstriction in animals and in man. $J$. Exper. Med., 1926, 43, 31.

13. Goldring, W., and Chasis, H., Hypertension and Hypertensive Disease. New York, The Commonwealth Fund, 1944.

14. Phillips, R. A., Van Slyke, D. D., Hamilton, P. B., Dole, V. P., Emerson, K., Jr., and Archibald, R. M,. Measurement of specific gravities of whole blood and plasma by standard copper sulfate solutions. J. Biol. Chem., 1950, 183, 305.
15. Böger, A., and Wezler, K., Die Bestimmung des arteriellen Gesamtwiderstandes am Menschen. Arch. f. exper. Path. u. Pharmakol., 1937, 186, 43.

16. Kunkel, H. G., and Tiselius, A., Electrophoresis of proteins on filter paper. J. Gen. Physiol., 1951, 35, 89.

17. Gentzkow, C. J., An accurate method for the determination of blood urea nitrogen by direct Nesslerization. J. Biol. Chem., 1942, 143, 531.

18. Smith, H. W., The Kidney, Structure and Function in Health and Disease. New York, Oxford Univ. Press, 1951.

19. Brun, C., Knudsen, E. O. E., and Raaschou, F., The influence of posture on the kidney function. II. Glomerular dynamics in the passive erect posture. Acta med. Scandinav., 1945, 122, 332.

20. Smith, H. W., Goldring, W., Chasis, H., and Bradley, S. E., Unpublished observations.

21. Oliver, J., Architecture of the Kidney in Chronic Bright's Disease. New York, Paul B. Hoeber, Inc., 1939.

22. Addis, T., Barrett, E., Boyd, R. I., and Ureen, H. J., Renin proteinuria in the rat. $I$. The relation between the proteinuria and the pressor effect of renin. J. Exper. Med., 1949, 89, 131.

23. Luetscher, J. A., Jr., Electrophoretic analysis of plasma and urinary proteins. J. Clin. Invest., 1940, 19, 313. 